\title{
Panax ginseng extract rich in ginsenoside protopanaxatriol offers combinatorial effects in nitric oxide production via multiple signaling pathways
}

Hee Yoon Ahn ${ }^{1}$, So Young Hong ${ }^{1}$, Ji Yeon Kim² and Oran Kwon ${ }^{1 *}$

\begin{abstract}
The root of Panax ginseng C.A. Meyer has been shown to induce nitric oxide (NO) release resulting in a hypotensive effect. However, the main active component contributing to vascular endothelium relaxation remains uncertain. In this study, we hypothesized that multiple components of ginseng extract might have combinatory effects providing greater health benefits than a single ginsenosides. To test this hypothesis, we compared the NO-releasing and endothelial NO synthase (eNOS) activating potency of wide range of ginseng extracts (crude extract, CE; protopanaxatriol-enriched extract, TE; protopanaxadiol-enriched extract, DE) and individual ginsenosides (Rg1, Re and $\mathrm{Rb} 1$ ) in human umbilical vein endothelial cells. We found that TE had the highest potency in NO production, followed by $\mathrm{CE}, \mathrm{DE}$, and $\mathrm{Rg} 1$. We also observed that TE-treatment resulted in rapid activation of intracellular signaling pathways, immediate linear rise of NO, and increased eNOS activation. TE-induced activation of eNOS was abolished by pretreatment with wortmannin (inhibitor for PI3K-Akt), compound C (inhibitor for AMP activated protein kinase, AMPK) or L-NAME (inhibitor for NOS), whereas Rg1-induced eNOS phosphorylation was only partially attenuated. Further analysis revealed that TE, but not Rg1, results in AMPK phosphorylation at Thr ${ }^{172}$. These novel finding add evidence that the multiple components of Panax ginseng extract rich in protopanaxatriol offers combinatorial effects in NO production and vascular endothelium relaxation via multiple signaling pathways.
\end{abstract}

Keywords: PI3K/Akt, AMP activated protein kinase, Nitric oxide and human umbilical vein endothelial cells

\section{Background}

Ginseng, the root of Panax ginseng C.A. Meyer, has been widely used as both a medicine and a food in Asia for thousands of years (Helms 2004). Recently, there has been a renewed interest in investigating the health benefits of ginseng as well as its constituents by using modern techniques. Numerous studies have reported that ginseng functions as a free radical scavenger (Kang et al. 2006; Kitts and $\mathrm{Hu} 2000$ ) and an immunomodulator (Lee et al. 2005), contributing towards maintaining optimal health against certain chronic disease states and aging (Kitts and Hu 2000). More specifically, it has been demonstrated that ginseng had a potency to reduce

\footnotetext{
* Correspondence: orank@ewha.ac.kr

${ }^{1}$ Department of Nutritional Science and Food Management, Ewha Womans University, Seoul 120-750, Republic of Korea

Full list of author information is available at the end of the article
}

blood pressure by regulating vascular tone through induction of nitric oxide (NO) release in endothelial cells (Gillis 1997). Production of NO has been known to be induced by calcium-dependent endothelial nitric oxide synthase (eNOS), whose activity is regulated under various circumstances (Hien et al. 2010; Edirisinghe et al. 2008).

To date, more than hundred of ginsenosides has been identified from Araliaceae family (Jia and Zhao 2009a, Jia et al. 2009b) and are classified into two categories based on the presence or absence of a carboxyl group at the C-6 position; protopanaxadiols (PPDs) (e.g. Rb1, $\mathrm{Rb} 2, \mathrm{Rc}, \mathrm{Rd}, \mathrm{Rg} 3$ and $\mathrm{Rh} 2$ ) and protopanaxatriols (PPTs) (e.g. Re, Rf, Rg1, Rg2 and Rh1), respectively (Gillis, 1997). Typically, researchers have elucidated the mechanism of action of ginseng by treating human endothelial cells with highly purified individual ginsenosides. Leung 
et al. (Leung et al. 2007a, b) found that Rg1 and Re act as functional ligands for the glucocorticoid receptor, leading to rapid $\mathrm{NO}$ production. $\mathrm{Yu}$ et al. (Yu et al. 2007) reported that Rb1 induces NO production via androgen receptor-mediated eNOS phosphorylation. Hien et al. (Hien et al. 2010) investigated effects of Rg3 on endothelial NO production. Despite this large array of data for individual ginsenosides, the main active ginseng component contributing to vascular endothelium relaxation still remains uncertain.

In addition, since different ginsenosides produce differing effects, it has long been assumed that multiple components in ginseng extract can provide greater health benefits than a single ginsenoside (Kim and Kwon 2011; Low 2006). However, the combinatorial effect of multiple ginseng components in ginseng extract on NO production has not been well studied. Therefore, we investigated the study to compare ginseng extracts and individual ginsenosides for inducing $\mathrm{NO}$ production in human endothelial cells. To test this aim, a wide range of samples were prepared, including crude extract (CE), PPT-enriched extract (TE), PPD-enriched extract (DE) and single ginsenosides. Furthermore, to provide mechanistic explanations, we also compared the impacts of a selected extract and an equivalent amount of single ginsenoside ( $\mathrm{TE} v s \mathrm{Rg} 1$ ) on the activation of signaling pathways by using inhibitors.

\section{Results and discussion}

\section{Comparison of NO producing ability}

In our previous study, we demonstrated that administration of TE stimulated eNOS activation, enhanced NO production, improved vessel wall thickening, and alleviated hypertension in spontaneously hypertensive rats (Hong et al. 2012). In this study, as part of our continuous effort to test whether the presence of multiple active ginseng components may exert combinatorial effects, we compared the NO producing ability of a wide range of samples (Figure 1).

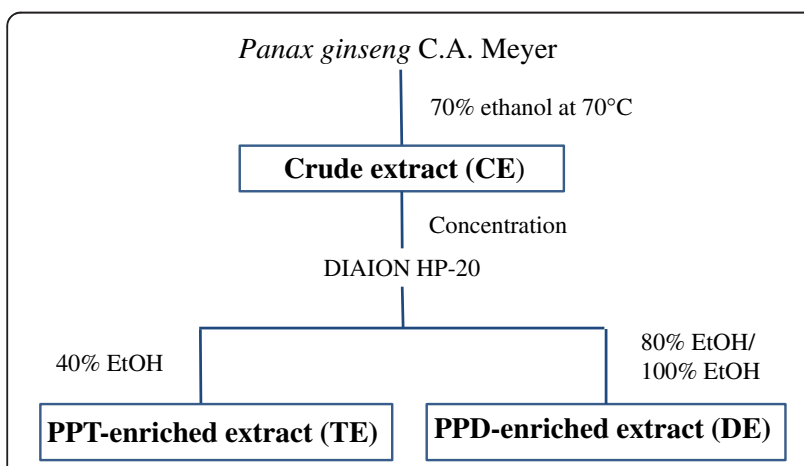

Figure 1 Schematic flow diagram of the ginseng extract preparation.
First, comparison of the intracellular bio-imaging of NO was performed by using 4,5-diaminofluorescein diacetate (DAF-2DA). Human umbilical vein endothelial cells (HUVECs) were treated with each sample $(150 \mu \mathrm{g} /$ $\mathrm{mL}$ of $\mathrm{CE}$, TE, or DE contains $25.8 \mu \mathrm{M}$ of $\mathrm{Rg} 1,31.1 \mu \mathrm{M}$ of $\operatorname{Re}$ or $52 \mu \mathrm{M}$ of Rb1, respectively.) for $10 \mathrm{~min}$, fixed, and then viewed by a fluorescence microscope. Sample concentrations were determined based on our previous in vivo study (Hong et al. 2012) and bioavailability of ginsenosides (Feng et al. 2010); Rg1 and Re concentrations were equivalent to those found in $\mathrm{TE}$ and $\mathrm{Rb} 1$ concentration was equivalent to those found in DE. Membrane permeable DAF-2 DA is taken-up by the cells and hydrolyzed by cellular esterase to form the membrane-impermeable compound 4,5-diaminofluorescein (DAF-2). As shown in Figure 2A, we observed a marked increase in intracellular DAF-2 in cells treated with TE. An increase was observed in cells treated with $\mathrm{CE}, \mathrm{DE}$ and Rg1. However, little DAF-2 production was detected in cells treated with Re or Rb1.

Broillet et al. (Broillet et al. 2001) questioned whether real-time biological detection of NO concentration is really directly correlated with $\mathrm{NO}$ release. Therefore, to confirm our results, we measured extracellular NO release from HUVECs. Consistent with increased NO production in the cell, we detected a significant increase in DAF-2 fluorescence intensity in the extracellular media in response to $\mathrm{TE}>\mathrm{CE}>\mathrm{DE}>\operatorname{Rg} 1$ compared to the control (Figure 2B). In contrast, Re and Rb1 treatment had no significant effect on NO release from the endothelial cells. These results support our hypothesis that multiple components in ginseng extract are more potent in inducing NO production than single ginsenosides, implicating the combinatorial interactions of these compounds. However, it should be noted that TE showed greater potency than $\mathrm{CE}$ and $\mathrm{DE}$. This might be attributed to the lower concentration of each active ginsenoside in $\mathrm{CE}$ or the differential effects of PPTs on the production of NO. For individual ginsenosides, Kang et al. (Kang et al. 1995) reported that $\operatorname{Rg} 1$ or $\operatorname{Re}$ treatment induced endothelium-dependent relaxation in rat aortas, whereas $\mathrm{Rb} 1$ or Rc treatment did not.

Ginsenosides are amphipathic in nature. Thus, they can directly interact with specific membrane proteins, triggering intracellular responses (Yue et al. 2007) and/ or can traverse cell membranes and bind nuclear receptors primarily affecting mRNA transcription and, subsequently, protein synthesis (Attele et al. 1999). While transcriptional effects with subsequent modification of protein expression requires hours to days to occur (Russell et al. 2000), we found that TE exposure at $150 \mu \mathrm{g} /$ $\mathrm{mL}$ concentration led to an linear increase in NO production and a plateau after 5 min (Figure 3), suggesting $\mathrm{TE}$-induced NO production is mediated by rapid 



Figure 2 Effect of ginseng extracts and individual ginsenosides on NO production in HUVECs. Confluent cells were incubated with $150 \mu \mathrm{g} / \mathrm{mL}$ ginseng extracts (CE, TE and DE), $26 \mu \mathrm{M}$ Rg1, $31 \mu \mathrm{M}$ Re, or $52 \mu \mathrm{M}$ Rb1 for 10 min. Bio-imaging of intracellular NO (A) and NO release (B) were measured using DAF-2 DA and DAF-2, respectively. Each bar represents mean \pm SD from three separate wells per condition. ${ }^{*} \mathrm{P}<0.05$ compared with control.

activation of intracellular signaling pathway. It should be stressed that HUVECs (Figure 3A) and the immortalized EA.hy926 cell line (Figure 3B) showed similar patterns in NO production in response to treatment, but basal NO production is higher in EA.hy926 cells compared to primary HUVECs. Thus, EA.hy926 cells were used for the subsequent experiments.

\section{Comparison of PI3K/Akt-mediated eNOS phosphorylation}

What intracellular signaling pathways are required for the TE-induced increase in NO production in endothelial cells? Accumulating evidence indicates that a number of protein kinases induce activation of eNOS by phosphorylating $\operatorname{Ser}^{1177}$ or $\mathrm{Thr}^{495}$ in endothelial cells. Based on previous studies (Chen et al. 1999), we focused on Akt- and AMP activated protein kinase (AMPK)- mediated phosphorylation of eNOS at Ser ${ }^{1177}$. TE and the equivalent amount of $\operatorname{Rg} 1$ were used for all subsequent experiments in the absence or presence of wortmannin (inhibitor of Akt signaling, $10 \mu \mathrm{M}$ ), compound $C$ (inhibitor of AMPK signaling, $10 \mu \mathrm{M}$ ), or NGnitro-L-arginine methyl ester (L-NAME) (inhibitor of NO synthase, $100 \mu \mathrm{M}$ ) in EA.hy926 cells.

Consistent with increased NO release, eNOS phosphorylation was observed in cells treated with $150 \mu \mathrm{g} / \mathrm{mL}$ TE or $25.8 \mu \mathrm{M} \operatorname{Rg} 1$ for $10 \mathrm{~min}$, and this effect was more pronounced in TE-treated cells $(p=0.01)$ than in Rg1-treated cells $(p=0.08)$, as shown in Figure 4 . It also showed that TE-induced eNOS phosphorylation was abolished by pretreatment with inhibitors for PI3K/Akt, AMPK or NO synthase (Figure 4A). In contrast, pretreatment of these inhibitors only partially attenuated Rg1-induced eNOS
(A)

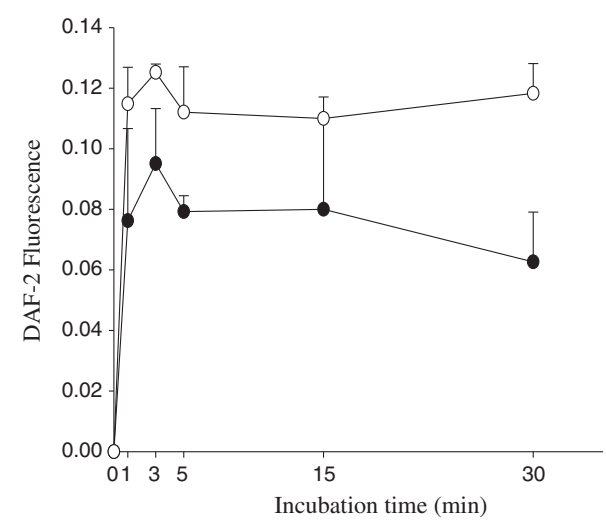

(B)

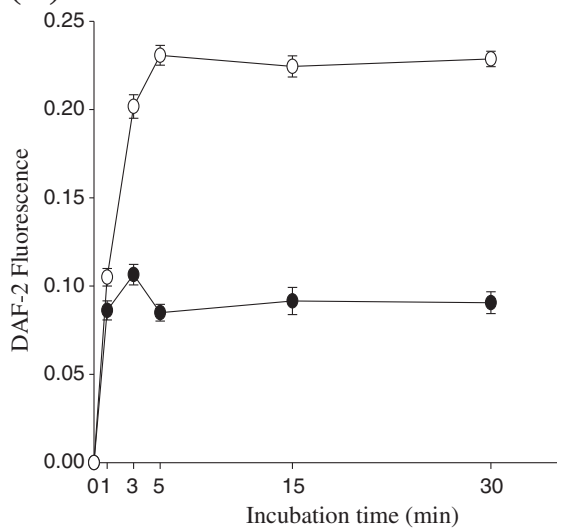

Figure 3 Time-course of TE-induced NO release in HUVECs (A) and EA.hy926 (B) cells. Cells were exposed to TE (O) or 1\% DMSO ( control) and time-dependent NO release was quantified at 0, 1, 3, 5, 15, and 30 min using DAF-2. Each point represents mean \pm SD from three separate wells per condition. 




(A)

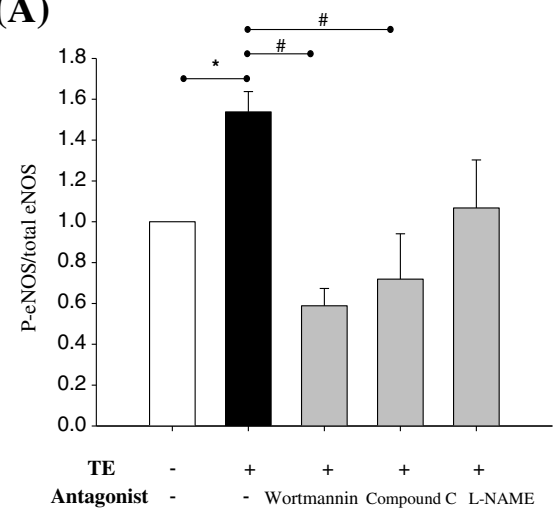



(B)



Figure 4 Inhibition of TE- or Rg1-induced eNOS activation in EA.hy926 cells. Confluent EA.hy926 cells were pretreated with wortmannin (PI3K/Akt inhibitor, $10 \mu \mathrm{M}$ ), compound C (AMPK inhibitor, $10 \mu \mathrm{M}$ ), or L-NAME (NOS inhibitor, $100 \mu \mathrm{M}$ ) for $30 \mathrm{~min}$, then treated with $150 \mu \mathrm{g} / \mathrm{mL}$ TE (A) or $26 \mu \mathrm{M} \mathrm{Rg} 1$ (B) for $10 \mathrm{~min}$. Representative western blots of intracellular eNOS or B-actin are presented. Quantitative analysis of optical density of eNOS (normalized to the density of $\beta$-actin) is shown at the bottom. Each bar represents mean \pm SD, from three separate wells per condition. ${ }^{*} \mathrm{P}<0.05$ compared with control. ${ }^{\#} \mathrm{P}<0.05$ compared with TE stimulation without inhibitor.

phosphorylation (Figure 4B). One interpretation of these data is that the stronger signals induced by TE treatment is attributed to the activation of multiple signaling pathways (Attele et al. 1999). Consistent with our results, several lines of evidence have demonstrated that Rg1 plays a role in PI3K/Akt-mediated eNOS phosphorylation leading to NO production in endothelial cells (Leung et al. 2006). In our previous work, we also demonstrated that TE activated eNOS phosphorylation via the activation of Akt in rats (Hong et al. 2012).

\section{Comparison of AMPK-mediated eNOS phosphorylation}

However, there is lack of information concerning the role of ginsenosides in relation to AMPK-mediated phosphorylation of eNOS. To dissect the signaling pathway required for phosphorylation of AMPK at $\mathrm{Thr}^{172}$ and subsequent phosphorylation of eNOS, we treated EA. hy926 cells with TE or Rg1 in the presence or absence of various inhibitors. Figure 5 showed that phosphorylation of AMPK markedly decreased below control level by pretreatment with compound $\mathrm{C}$ in both TE-treated $(p=0.02)$ and Rg1-treated $(p=0.08)$ cells, demonstrating noticeable inhibition of constitutive activation of AMPK. Interestingly, the result also revealed that there was a tendency to increase the phosphorylation of AMPK by TE treatment $(\mathrm{p}=0.17)$ (Figure $5 \mathrm{~A})$, whereas $\operatorname{Rg} 1$ treatment did not affect AMPK activation (Figure 5B). As for the effect of ginsenosides on the phosphorylation of AMPK, recently,
Hien et al. (2010) demonstrated AMPK-dependent eNOS phosphorylation in Rg3-treated endothelial cells. They also showed that Rg3-stimulated eNOS phosphorylation was reversed by AMPK inhibition. However, no report was found in the literature regarding the effect of Rg1 on AMPK-mediated eNOS phosphorylation.

\section{Conclusions}

Our results clearly demonstrate that TE, a PPT-enriched ginseng extract, is superior in inducing $\mathrm{NO}$ production, compared to $\mathrm{CE}, \mathrm{DE}$, or individual ginsenosides in human endothelial cells. The stronger ability of TE to induce NO production is likely attributed to activation of multiple signal pathways, including Akt- and AMPKmediated phosphorylation of eNOS. The novel findings of this study provide additional evidence that the diverse array of PPTs in TE likely provides better health benefits via combinatorial interactions to stimulate multiple signaling pathways. Importantly, the present study was conducted with the consideration of ginsenosides only, given that the NO production potency of ginseng are attributed to ginsenosides; therefore the results reported here may provide limited insight on the potency of nonginsenoside constituents of ginseng. However, the present study may serve as a strategy to find the most appropriate preparation for plant extracts to achieve the maximum health benefits and to understand their role. 


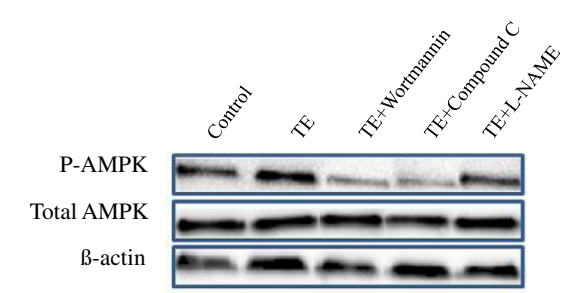

(A)

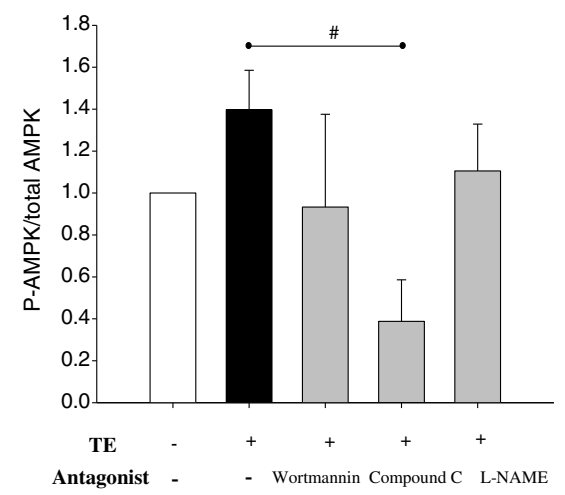

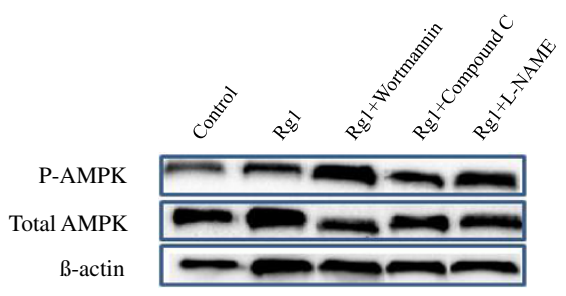

(B)

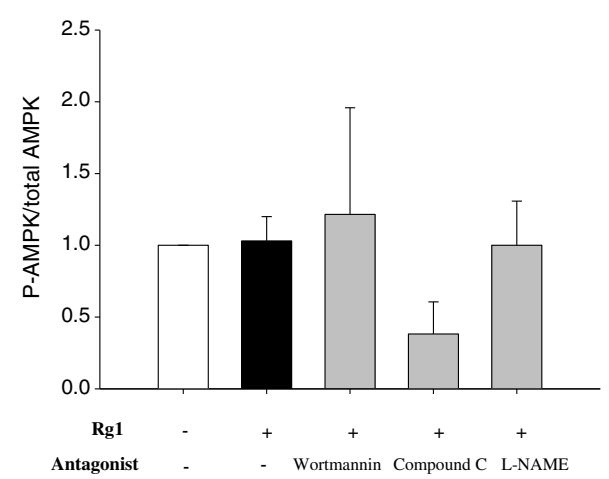

Figure 5 Inhibition of TE- or Rg1-induced AMPK activation in EA.hy926 cells. Confluent EA.hy926 cells were pretreated with wortmannin (PI3K Akt inhibitor, $10 \mu \mathrm{M}$ ), compound C (AMPK inhibitor, $10 \mu \mathrm{M}$ ), or L-NAME (NOS inhibitor, $100 \mu \mathrm{M}$ ) for $30 \mathrm{~min}$, then treated with $150 \mu \mathrm{g} / \mathrm{mL}$ TE (A) or $26 \mu \mathrm{M} \mathrm{Rg1} \mathrm{(B)} \mathrm{for} 10 \mathrm{~min}$. Representative western blots of intracellular AMPK or B-actin are presented. Quantitative analysis of optical density of AMPK (normalized to the density of $\beta$-actin) is shown at the bottom. Each bar represents mean \pm SD, from three separate wells per condition. ${ }^{\#}$ P 0.05 compared with TE stimulation without inhibitor.

\section{Methods Reagents}

Ginsenoside Re, Rb2, Rc, Rd, Rg1 and Rb1 were purchased from ChromaDex (Irvine, CA, USA). L-NAME (NO synthase inhibitor) and compound C (AMPK inhibitor) were purchased from Cayman Chemical (Ann Arbor, MI, USA). Wortmannin (PI3K-Akt inhibitor) was purchased from Sigma (St. Louis, MO, USA). Antibodies (eNOS, phospho-eNOS ${ }^{\text {Ser 1177; }}$ AMPK, and AMPK ${ }^{\text {Thr172 }}$ ) were purchased from Cell Signaling Technology (Beverly, MA, USA). HUVECs, the immortalized HUVEC cell line EA.hy 926 and culture medium were purchased from the American Type Culture Collection (Bethesda, MD, USA). DAF-2 and DAF-2 DA were purchased from Alexis Biochemicals (Grünberg, Germany) and Cayman Chemical, respectively.

\section{Preparation of ginseng extracts}

The CE, TE and DE were kindly provided by CJ Cheiljedang Corp. (Seoul, Korea). Briefly, dried ginseng (Panax ginseng C.A. Meyer) roots were cut into small pieces and refluxed in 70\% ethanol. After removing ethanol, CEs were eluted on DIAION HP-20 ion exchange resin (Mitsubishi Chemical Co., Tokyo, Japan) to obtain TE and DE by $40 \%$ ethanol elution and by both $100 \%$ and $80 \%$ ethanol elution, respectively (Figure 1). The yields were found to be $50 \%, 1 \%$ and $5 \%$ for CE, TE and $\mathrm{DE}$, respectively. The major ginsenosides in freeze-dried extracts were quantified by comparison with standards using an Agilent 1100 HPLC system (Palo Alto, CA, USA) equipped with a reversed-phase column (Venusil XBP C18, 250 X 4.6 mm, i.d. $5 \mu \mathrm{m}$, Agela Technology, Newark, DE, USA) (Table 1).

Table 1 Content of major ginsenosides in test materials

\begin{tabular}{|c|c|c|c|c|c|c|}
\hline \multirow[t]{2}{*}{ Preparations } & \multicolumn{2}{|c|}{ Protopanaxatriol type } & \multicolumn{4}{|c|}{ Protopanaxadiol type } \\
\hline & $\operatorname{Rg} 1(\mathrm{mg} / \mathrm{g})$ & $\operatorname{Re}(\mathrm{mg} / \mathrm{g})$ & $\mathrm{Rb} 1$ (mg/g) & $\mathrm{Rb2}(\mathrm{mg} / \mathrm{g})$ & $\mathrm{Rc}(\mathrm{mg} / \mathrm{g})$ & $\mathrm{Rd}(\mathrm{mg} / \mathrm{g})$ \\
\hline Crude extract (CE) & 5.9 & 18.2 & 25 & 13.5 & 27.3 & 9.6 \\
\hline PPT-enriched extract (TE) & 136.7 & 325.6 & 63.1 & 29.2 & 73.2 & 11.5 \\
\hline PPD-enriched extract (DE) & 14.1 & 26.9 & 229.9 & 132.2 & 261.2 & 81.3 \\
\hline
\end{tabular}




\section{Cell culture and treatments}

For NO production assay, confluent cells in 12-well plates were serum-starved overnight and treated with the respective samples in $\mathrm{Ca}^{+2}$-containing phosphate buffered saline for $10 \mathrm{~min}$ at $37^{\circ} \mathrm{C}$. For inhibitor assays, confluent cells in $100 \mathrm{~mm}$ dishes were serum-starved overnight, pretreated with different inhibitors (L-NAME, $100 \mu \mathrm{M}$; wortmannin, $10 \mu \mathrm{M}$; compound $\mathrm{C}, 10 \mu \mathrm{M}$ ) for $30 \mathrm{~min}$, and then treated with TE or Rg1 for $10 \mathrm{~min}$. Ginseng extracts and ginsenosides were prepared fresh by diluting a 100 -fold concentrated stock solution prepared in dimethyl sulfoxide.

\section{Measurement of intracellular and extracellular NO production}

For intracellular NO production, confluent cells were pre-incubated with $5 \mu \mathrm{M}$ DAF-2 DA for $30 \mathrm{~min}$ at $37^{\circ} \mathrm{C}$ in darkness, rinsed with fresh suspension buffer to remove excess fluorophore, and treated with the respective samples for $10 \mathrm{~min}$. The cells were fixed in $2 \%$ paraformaldehyde and green fluorescence zimages obtained using a fluorescent microscope (Nikon ECLIPSE TS 100, Nikon, Tokyo, Japan) at $495 \mathrm{~nm}$ excitation and $515 \mathrm{~nm}$ emission wavelength (Kojima et al. 1998). For extracellular NO release, DAF-2 $(1 \mu \mathrm{M})$ was added in assay medium for $5 \mathrm{~min}$ at $37^{\circ} \mathrm{C}$ in darkness after treatment with respective samples. Aliquots of the solutions were sampled and fluorescence was measured using a Thermo Scientific Fluorometer (Barrington, IL, USA) at $495 \mathrm{~nm}$ excitation and $515 \mathrm{~nm}$ emission wavelength (Leikert et al. 2001).

\section{Western blot analysis}

Cells were stimulated with respective samples for $10 \mathrm{~min}$ and then lysed in lysis buffer. Equal quantities of protein were resolved by SDS-polyacrylamide gel electrophoresis and transferred onto polyvinylidene difluoride membranes (Bio-Rad, Hercules, CA, USA). The proteins were probed with the indicated primary antibodies, and then incubated either goat anti-rabbit or goat anti-mouse secondary antibody. Bands were visualized using the West-one Western Blot Detection System (iNtRON Biotechnology, Korea). Band intensity was quantified using ChemiDoc XRS + Systems with Image Lab software (Bio-Rad, Hercules, CA, USA) and normalized to $\beta$-actin (Santa Cruz Biotechnology) densitometric values.

\section{Statistical analysis}

All data shown are representative of at least three experiments that yielded similar results. Data are presented as the mean of triplicate samples with error bars indicative of the standard deviations. The numerical results were analyzed using one-way analysis of variance with post hoc Dunnett's multiple range tests. $P<0.05$ was considered statistically significant. Statistical analyses were performed using the SAS package version 9.2 (SAS Institute, Cary, NY).

\section{Abbreviations}

AMPK: AMP activated protein kinase; CE: Crude extract; DAF-2: 4,5diaminofluorescein; DAF-2DA: DAF-2 diacetate; DE: PPD-enriched extract; eNOS: Endothelial nitric oxide synthase; HUVECs: Human umbilical vein endothelial cells; L-NAME: NG-nitro-L-arginine methyl ester; NO: Nitric oxide; PPD: Protopanaxadiol; PPT: Protopanaxatriol; TE: PPT-enriched extract.

\section{Competing interests}

The authors declare that they have no competing interest.

\section{Authors' contributions}

HYA and SYH performed the experiments and data analysis; HYA, JYK and OK contributed to study design and manuscript writing; and OK had primary responsibility for final content. All authors read and approved the final manuscript.

\section{Acknowledgements}

We thank the staff of CJ Cheiljedang Corp. (Seoul, Korea) for preparation of ginseng extracts. This project was supported by the Ministry of Knowledge \& Economy (National Platform, Project B0009639) and the Ministry of Education, Science, and Technology (Brain Korea 21, Project 2006-0519-4-7).

\section{Author details}

'Department of Nutritional Science and Food Management, Ewha Womans University, Seoul 120-750, Republic of Korea. ${ }^{2}$ Department of Food Science and Technology, Seoul National University of Science and Technology, Seoul 139-743, Republic of Korea.

Received: 15 January 2013 Accepted: 27 February 2013

Published: 9 March 2013

\section{References}

Attele AS, Wu JA, Yuan CS (1999) Ginseng pharmacology: multiple constituents and multiple actions. Biochem Pharmacol 58:1685-1693

Broillet M, Randin O, Chatton J (2001) Photoactivation and calcium sensitivity of the fluorescent NO indicator 4,5-diaminofluorescein (DAF-2): implications for cellular NO imaging. FEBS Lett 491:227-232

Chen ZP, Mitchelhill Kl, Michell BJ et al (1999) AMP-activated protein kinase phosphorylation of endothelial NO synthase. FEBS Lett 443:285-289

Edirisinghe I, Burton-Freeman B, Varelis P, Kappagoda T (2008) Strawberry extract caused endothelium-dependent relaxation through the activation of PI3 kinase/Akt. J Agric Food Chem 56:9383-9390

Feng L, Wang L, Hu C, Jiang X (2010) Pharmacokinetics, tissue distribution, metabolism, and excretion of ginsenoside Rg1 in rats. Arch Pharm Res 33:1975-1984

Gillis CN (1997) Panax ginseng pharmacology: a nitric oxide link? Biochem Pharmacol 54:1-8

Helms S (2004) Cancer prevention and therapeutics: panax ginseng. Altern Med Rev 9:259-274

Hien TT, Kim ND, Pokharel YR et al (2010) Ginsenoside Rg3 increases nitric oxide production via increases in phosphorylation and expression of endothelial nitric oxide synthase: essential roles of estrogen receptor-dependent PI3-kinase and AMP-activated protein kinase. Toxicol Appl Pharmacol 246:171-183

Hong SY, Kim JY, Ahn HY, Shin JH, Kwon O (2012) Panax ginseng extract rich in ginsenoside protopanaxatriol attenuates blood pressure elevation in spontaneously hypertensive rats by affecting the Akt-dependent phosphorylation of endothelial nitric oxide synthase. J Agric Food Chem 60:3086-3091

Jia L, Zhao Y (2009a) Current evaluation of the millennium phytomedicine ginseng (I): etymology, pharmacognosy, phytochemistry, market and regulations. Curr Med Chem 16(19):2475-2484

Jia L, Zhao Y, Liang XJ (2009b) Current evaluation of the millennium phytomedicine - ginseng (II): collected chemical entities, modern pharmacology, and clinical applications emanated from traditional Chinese medicine. Curr Med Chem 16(22):2924-2942 
Kang SY, Schini-Kerth VB, Kim ND (1995) Ginsenosides of the protopanaxatriol group cause endothelium-dependent relaxation in the rat aorta. Life Sci 56:1577-1586

Kang KS, Kim HY, Pyo JS, Yokozawa T (2006) Increase in the free radical scavenging activity of ginseng by heat-processing. Biol Pharm Bull 29:750-754

Kim JY, Kwon O (2011) Culinary plants and their potential impact on metabolic overload. Ann N Y Acad Sci 1229:133-139

Kitts D, Hu C (2000) Efficacy and safety of ginseng. Public Health Nutr 3:473-485

Kojima H, Nakatsubo N, Kikuchi K et al (1998) Direct evidence of NO production in rat hippocampus and cortex using a new fluorescent indicator: DAF-2 DA. Neuroreport 9:3345-3348

Lee TK, Johnke RM, Allison RR, O'Brien KF, Dobbs LJ (2005) Radioprotective potential of ginseng. Mutagenesis 20:237-243

Leikert JF, Räthel TR, Müller C, Vollmar AM, Dirsch VM (2001) Reliable in vitro measurement of nitric oxide released from endothelial cells using low concentrations of the fluorescent probe 4,5-diaminofluorescein. FEBS Lett 506:131-134

Leung KW, Pon YL, Wong RN, Wong AS (2006) Ginsenoside-Rg1 induces vascular endothelial growth factor expression through the glucocorticoid receptorrelated phosphatidylinositol 3-kinase/Akt and beta-catenin/T-cell factordependent pathway in human endothelial cells. J Biol Chem 281:36280-36288

Leung KW, Cheung LW, Pon YL et al (2007a) Ginsenoside Rb1 inhibits tube-like structure formation of endothelial cells by regulating pigment epithelium-derived factor through the oestrogen beta receptor. Br J Pharmacol 152:207-215

Leung KW, Leung FP, Huang Y, Mak NK, Wong RN (2007b) Non-genomic effects of ginsenoside-Re in endothelial cells via glucocorticoid receptor. FEBS Lett 581:2423-2428

Low DT (2006) A reason to season: the therapeutic benefits of spices and culinary herbs. Explore (NY) 2:446-449

Russell KS, Haynes MP, Sinha D, Clerisme E, Bender JR (2000) Human vascular endothelial cells contain membrane binding sites for estradiol, which mediate rapid intracellular signaling. Proc Natl Acad Sci USA 97:5930-5935

Yu J, Eto M, Akishita M, Kaneko A, Ouchi Y, Okabe T (2007) Signaling pathway of nitric oxide production induced by ginsenoside Rb1 in human aortic endothelial cells: a possible involvement of androgen receptor. Biochem Biophys Res Commun 353:764-769

Yue PY, Mak NK, Cheng YK et al (2007) Pharmacogenomics and the Yin/Yang actions of ginseng: anti-tumor, angiomodulating and steroid-like activities of ginsenosides. Chin Med. doi:10.1186/1749-8546-2-6

doi:10.1186/2193-1801-2-96

Cite this article as: Ahn et al:: Panax ginseng extract rich in ginsenoside protopanaxatriol offers combinatorial effects in nitric oxide production via multiple signaling pathways. SpringerPlus 2013 2:96.

\section{Submit your manuscript to a SpringerOpen ${ }^{\circ}$ journal and benefit from:}

- Convenient online submission

Rigorous peer review

- Immediate publication on acceptance

- Open access: articles freely available online

- High visibility within the field

- Retaining the copyright to your article

Submit your next manuscript at $>$ springeropen.com 